\title{
BMJ Open Comparison and validation of International Consensus Diagnostic Criteria for diagnosis of autoimmune pancreatitis from pancreatic cancer in a Taiwanese cohort
}

\author{
Ming-Chu Chang, ${ }^{1}$ Po-Chin Liang, ${ }^{2}$ I-Shiow Jan, ${ }^{3}$ Ching-Yao Yang, ${ }^{4}$ Yu-Wen Tien, ${ }^{4}$ \\ Shu-Chen Wei, ${ }^{1}$ Jau-Min Wong, ${ }^{1}$ Yu-Ting Chang ${ }^{1}$
}

To cite: Chang M-C, Liang P-C, Jan I-S, et al. Comparison and validation of International Consensus Diagnostic Criteria for diagnosis of autoimmune pancreatitis from pancreatic cancer in a Taiwanese cohort. BMJ Open 2014;4:e005900. doi:10.1136/bmjopen-2014005900

- Prepublication history for this paper is available online. To view these files please visit the journal online (http://dx.doi.org/10.1136/ bmjopen-2014-005900).

Received 12 June 2014 Revised 12 July 2014 Accepted 18 July 2014

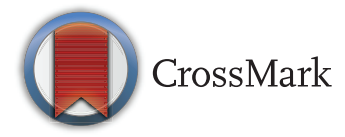

For numbered affiliations see end of article.

Correspondence to Dr Yu-Ting Chang; yutingchang@ntu.edu.tw

\section{ABSTRACT}

Objectives: The International Consensus Diagnostic Criteria (ICDC) designed to diagnosis autoimmune pancreatitis (AIP) has been proposed recently. The diagnostic performance of ICDC has not been previously evaluated in diffuse-type and focal-type AIP, respectively, in comparison with the revised HISORt and Asian criteria in Taiwan.

Design: Prospective, consecutive patient cohort. Setting: Largest tertiary referred centre hospital managing pancreatic disease in Taiwan.

Participants: 188 patients with AIP and 130 with tissue proofed pancreatic adenocarcinoma were consecutively recruited.

Interventions: The ICDC, as well as revised HISORt and Asian criteria, was applied for each participant. Each diagnostic criterion of ICDC was validated with special reference to levels 1 and 2 in diffuse-type and focal-type AIP.

Outcomes: Sensitivity, specificity and accuracy. Each diagnostic criterion of ICDC was validated with special reference to levels 1 and 2 in AIP and focaltype AIP.

Results: The sensitivity, specificity and accuracy of ICDC for all AIP were the best: $89.4 \%, 100 \%$ and $93.7 \%$, respectively, in these three criteria. The sensitivity, specificity and accuracy of ICDC for focaltype AIP (84.9\%, 100\% and 93.8\%) were also the best among these three criteria. The area under the curve of receiver-operator characteristic of ICDC was 0.95 (95\% $\mathrm{Cl} 0.92$ to 0.97$)$ in all AIP and $0.93(95 \% \mathrm{Cl} 0.88$ to 0.97) in focal-type AIP.

Conclusions: The sensitivity, specificity and accuracy of ICDC are higher than the revised HISORt and Asian criteria. The sensitivity, specificity and accuracy of each criterion are higher in diffuse-type AIP compared with focal-type AIP. Under the same specificity, the sensitivity and accuracy of ICDC are higher than other diagnostic criteria in focal-type AIP. ICDC has better diagnostic performance compared with previously proposed diagnostic criteria in diffuse-type and focaltype AIP.

\section{Strengths and limitations of this study}

- This is the first study to determine the diagnostic accuracy of International Consensus Diagnostic Criteria (ICDC) of autoimmune pancreatitis (AIP) from pancreatic cancer with focus on 'focal'-type AIP in Taiwan.

- The study focuses only on type 1 AIP in our study because the prevalence of type 2 AIP is relatively low in eastern countries including Taiwan. The role of ICDC in type 2 AIP needs further study.

- The diagnostic performance of ICDC compared with other diagnostic criteria proposed in other regions or countries, other than the revised HISORt and Asian criteria, are needed to confirm the universalisation of the diagnosis of AIP.

\section{INTRODUCTION}

Autoimmune pancreatitis (AIP) is a unique type of chronic pancreatitis characterised by elevated serum IgG4, swelling of the pancreas, irregular narrowing of the main pancreatic duct (MPD), histological evidence of lymphoplasmacytic inflammation and a good response to steroid therapy. ${ }^{1}$ Although some advance has been made in the diagnosis and treatment of AIP in past years, ${ }^{2}$ the diagnosis of AIP is still a great clinical challenge, especially in the differential diagnosis from pancreatic cancer (PC). ${ }^{3-5}$ Correct diagnosis of AIP could avoid delayed unnecessary resection of pancreas and vice versa, to avoid delayed treatment of PC. In 2002, the Japan Pancreas Society proposed diagnostic criteria for AIP based on imaging, serology and histology. At that time, the serological criteria included elevated $\gamma$-globulin, IgG and autoantibodies. ${ }^{6}$ In 2006, the revised Japanese criteria were modified and added IgG4 to the serological 
criteria. ${ }^{7}$ In 2008, the Asian diagnostic criteria was established by modification of the Japanese and Korean diagnostic criteria. ${ }^{8}$ In western countries, the HISORt criteria were proposed from America. ${ }^{9}$ In 2011, the International Consensus Diagnostic Criteria (ICDC) were proposed which classified AIP into types 1 and 2. Type 1 is featured histologically by lymphoplasmacytic sclerosing pancreatitis (LPSP) and type 2 by idiopathic duct-centric pancreatitis. The ICDC included five cardinal features of AIP including parenchymal imaging, ductal imaging, serology, other organ involvement (OOI), histology of pancreas and response to steroid therapy. Each criterion was further classified into two levels (levels 1 and 2). The aim of the proposal of ICDC was intended to improve the diagnosis of AIP. ${ }^{10}$ AIP could be also classified into focal-type and diffuse-type AIP according to the involvement of pancreatic enlargement. In clinical settings, it is more important and also difficult to differentiate focal-type AIP from PC. To date, it still lacks a simple parameter with absolute diagnostic value. Therefore, the use of combined parameters according to different diagnostic criteria in different countries exists.

The objective of this study is to evaluate the diagnostic performance (sensitivity, specificity and accuracy) of ICDC of AIP from differentiating PC in a prospectively collected cohort in Taiwan, ${ }^{11-13}$ compared with the two most commonly used criteria in our country before ICDC was made (revised HISORt and Asian criteria). The diagnostic role of each cardinal feature of ICDC will be compared with the revised HISORt and Asian criteria in diffuse-type and focal-type AIP, respectively.

\section{METHODS}

\section{Study participants}

Between January 1996 and December 2013, we consecutively collected 188 patients with AIP (95 men and 93 women) at the National Taiwan University Hospital, a tertiary referred centre and also the largest medical centre for management of pancreatic diseases in Taiwan. ${ }^{11}$ All patients with AIP fulfilled at least one of the HISORt criteria $(158 / 188,84 \%)$, or the Asian diagnostic criteria $(162 / 188,86.2 \%)$, or the ICDC criteria $(168 / 188$, $89.4 \%$ ) for AIP. All patients were type 1 AIP. All patients were followed up for at least 12 months. A total of 130 consecutive patients (65 men and 65 women) with cytological or/and pathologically confirmed adenocarcinoma of the pancreas were enrolled as a control group. The patients' mean age was 51.4 (range, 33-78 years) and 60.9 years (range 32-78 years), respectively, in patients with AIP and PC. All the patients' medical charts were reviewed and the patients' demographic data, including age, gender, serological studies, image studies and clinical manifestations, etc, were collected.

\section{Findings of diagnostic criteria of ICDC}

We categorised all patients with AIP and PC as level 1 findings, level 2 findings or neither for each of the five criteria (parenchymal imaging, ductal imaging, serology, other OOI, histology of pancreas and response to steroid therapy) according to ICDC. For parenchymal imaging, the frequencies of diffuse enlargement, focal enlargement and atypical imaging (pancreatic duct dilation or calcification or atrophy) were evaluated. Enlargement of the pancreas was defined as when the width of the pancreatic body or tail exceeds two-thirds of the transverse diameter of the vertebral body or if the width of the pancreatic head exceeds the full transverse diameter of the vertebral body. ${ }^{14}$ For ductal imaging, the MPD diameter was measured by the use of abdominal CT and/or MR cholangiopancreatography (MRCP) and/or endoscopic retrograde pancreatography. The frequencies of long stricture without marked upstream dilation, of multiple strictures without marked upstream dilation, of segmental/focal narrowing without marked upstream dilation and of marked upstream dilation of the MPD were evaluated. Pancreatic duct dilation was defined as the diameter of the MPD exceeding $5 \mathrm{~mm}$. Parenchymal and ductal imaging scans were analysed by three experts (M-CG, Y-TC and P-CL).

For OOI, the frequencies of segmental/multiple proximal bile duct stricture, retroperitoneal fibrosis, symmetrically enlarged salivary/lachrymal glands and radiological evidence of renal involvement were evaluated. Bile duct stricture was evaluated by MRCP or ERC, or percutaneous transhepatic cholangiography. Retroperitoneal fibrosis was evaluated by contrast enhanced CT or MRI. Symmetrically enlarged salivary/lachrymal glands were evaluated by physical examination or/and CT.

For histology criteria of pancreas in AIP, there were 25 patients received pancreatectomy. Six patients received a biopsy of the pancreas. Pancreatic histology was evaluated by an experienced pathologist who was blinded to the other data.

Regarding steroid therapy, 126 patients $(67 \%)$ received corticosteroid therapy as the initial treatment of AIP. The dose for induction therapy was started from 30 to $40 \mathrm{mg}$ / day for 2-4 weeks and then tapered $5 \mathrm{mg}$ /week gradually. The treatment response of steroid was nearly $98 \%$.

We evaluated of the frequencies of level 1 findings, level 2 finding and neither of each criterion in all patients with AIP and PC. Sensitivity, specificity and accuracy were compared between ICDC, revised HISORt criteria and Asian criteria. We also evaluated the cases which did not fit the diagnosis with AIP according to each criterion.

\section{Statistical analysis}

We calculated the sensitivity, specificity and accuracy of each diagnostic criterion. The between-group demographic data were compared by the Student unpaired $t$ test for continuous data and by the $\chi^{2}$ test for categorical data. Receiver-operator characteristic (ROC) curves and area under the curves (AUCs) were estimated after logistic regressions in different criteria in all AIP and focaltype AIP subgroups and presented with corresponding 
95\% CIs. Statistical calculations were carried out using SPSS V.17 statistical software (SAS Institute, Cary, North Carolina, USA). All reported $\mathrm{p}$ values were two-sided. Differences with a $\mathrm{p}$ value less than 0.05 were considered to be statistically significant.

\section{RESULTS}

\section{Parenchymal imaging}

Of the 188 patients, $90(50.5 \%)$ and $93(49.5 \%)$ with AIP were categorised as levels 1 and 2, respectively. All patients with PC were classified as level 2 (table 1).

\section{Ductal imaging}

Ductal imaging was evaluated by at least one of the ERC or MRCP in all patients. Among them, 93 (49.5\%) of 188 patients with AIP and no patients with PC were categorised as level 1 . There were $68(36.2 \%)$ of 188 patients with AIP and $13(10 \%)$ of 130 patients with PC were categorised as level 2 (table 1). Marked MPD dilation was observed to be significantly more frequent in patients with PC $(\mathrm{n}=117 ; 90 \%)$ than in those with AIP $(\mathrm{n}=27 ; 14.4 \%, \mathrm{p}<0.001)$. Among the 27 AIP patients with MPD dilation, narrowing of the downstream MPD was observed in 3 patients and the others with normal downstream appearance.

\begin{tabular}{|c|c|c|}
\hline Features & AIP $(n=188)$ & $P C(n=130)$ \\
\hline \multicolumn{3}{|c|}{ Parenchymal imaging } \\
\hline Level 1 & 95 (50.5\%) & $0.0(0.0 \%)$ \\
\hline Level 2 & $93(49.5 \%)$ & $130(100.0 \%)$ \\
\hline Level $1+2$ & $188(100.0 \%)$ & $130(100.0 \%)$ \\
\hline Non-level 1, 2 & $0(0.0 \%)$ & $0.0(0.0 \%)$ \\
\hline \multicolumn{3}{|l|}{ Ductal imaging } \\
\hline Level 1 & $93(49.5 \%)$ & $0.0(0.0 \%)$ \\
\hline Level 2 & $68(36.2 \%)$ & $13(10.0 \%)$ \\
\hline Level $1+2$ & $161(85.6 \%)$ & $13(10.0 \%)$ \\
\hline Non-level 1, 2 & 27 (14.4\%) & $117(90.0 \%)$ \\
\hline \multicolumn{3}{|l|}{ Serology } \\
\hline Level 1 & 47/128 (36.7\%) & 4/84 (4.8\%) \\
\hline Level 2 & $55 / 128(42.9 \%)$ & 3/84 (3.6\%) \\
\hline Level $1+2$ & $102 / 128(79.7 \%)$ & $7 / 84(8.3 \%)$ \\
\hline Non-level 1, 2 & $26 / 128(20.3 \%)$ & 77/84 (91.7\%) \\
\hline \multicolumn{3}{|l|}{$\mathrm{OOI}$} \\
\hline Level 1 & $63(33.5 \%)$ & $0(0.0 \%)$ \\
\hline Level 2 & $64(34.0 \%)$ & $0(0.0 \%)$ \\
\hline Level $1+2$ & $127(92.5 \%)$ & $0(0.0 \%)$ \\
\hline Non-level 1, 2 & $61(32.4 \%)$ & $130(100.0 \%)$ \\
\hline \multicolumn{3}{|c|}{ Histology of the pancreas } \\
\hline Level 1 & $28(14.9 \%)$ & $0(0.0 \%)$ \\
\hline Level 2 & $3(1.6 \%)$ & $0(0.0 \%)$ \\
\hline Level $1+2$ & $31(16.5 \%)$ & $0(0.0 \%)$ \\
\hline Non-level 1, 2 & $157(83.5 \%)$ & $130(100.0 \%)$ \\
\hline
\end{tabular}

AIP, autoimmune pancreatitis; ICDC, International Consensus Diagnostic Criteria; OOI, other organ involvement; PC, pancreatic cancer.

\section{Serology}

Forty-seven (36.7\%) of 188 patients with AIP and 4 $(4.8 \%)$ of 84 patients with PC, respectively, were categorised as level 1(table 1).The mean serum IgG4 level was $346.6 \pm 56.2 \mathrm{mg} / \mathrm{dL}$, which was statistically significantly higher than that in patients with PC, 119.2 \pm 23.9 $\mathrm{mg} / \mathrm{dL}$. The frequencies of serum level above 280 (level 1) and $140 \mathrm{mg} / \mathrm{dL}$ (level 2) were significantly higher in patients with AIP $(\mathrm{p}<0.001$.)

\section{Other organ involvement}

Sixty-three $(33.5 \%)$ of 188 patients with AIP were categorised as level 1 and none of the 130 patients with PC were categorised as level 1 or 2 (table 1). Proximal bile duct stricture was observed in 53 patients $(28.2 \%)$ with AIP, and retroperitoneal fibrosis was observed in 5 patients $(2.7 \%)$ with AIP, both level 1 findings. Enlarged salivary/lacrimal glands were observed in 58 patients $(27.6 \%)$ with AIP and renal involvement in 9 patients $(4.8 \%)$; these met level 2 criteria. Neither enlarged salivary/lacrimal glands nor renal involvement was observed in any patients with PC.

\section{Histology of pancreas}

There were $31(16.4 \%)$ patients with AIP who were categorised as level 1 or 2 and none of the 130 patients with PC were categorised as level 1 or 2 (table 1). There were 28 patients $(14.9 \%)$ with level 1 evidence and 3 patients (1.6\%) with level 2 evidence in histological LPSP. No patients with PC fulfilled the level 1 or 2 criteria.

\section{Response to steroid therapy}

Of the 128 patients who received steroid treatment as the initial treatment (induction therapy), 126 (98.4\%) showed steroid response with improvement clinically, serologically and morphologically. Two of the patients with diffuse pancreatic enlargement and narrowing of the MPD received steroids but there was no morphological response. Endoscopic ultrasound-guided fineneedle aspiration (EUS-FNA) was performed but no malignancy was detected. These two patients discontinued steroid use after 3-month treatment and were followed up regularly for 18 and 20 months, respectively, but no malignancy was documented, although the pancreatic enlargement did not subside.

\section{Diagnosis on the basis of ICDC and revised HISORt and Asian criteria}

Of the 188 patients with AIP, 116 patients were diagnosed as definite AIP; 35 patients were diagnosed as probable AIP and 17 were classified as not otherwise specified (NOS). Among these patients, the primary basis for diagnosis was histology in 31 patients, response to steroid therapy in 126 patients and imaging in 188 patients. There were 20 patients in this study deniable for type 1 AIP. All patients with PC were deniable for type 1 AIP on the basis of ICDC. The sensitivity, specificity and accuracy of ICDC for type 1 AIP were $89.4 \%$, 
Table 2 Comparison of diagnostic performance of different criteria for AIP from pancreatic cancer

\begin{tabular}{lllll}
\hline & Case number & Sensitivity (\%) & Specificity (\%) & Accuracy (\%) \\
\hline All AlP & & & & \\
Fit Asian & 162 & 86.2 & 100.0 & 91.8 \\
Fit revised HISORt & 158 & 84.0 & 100.0 & 90.5 \\
Fit ICDC & 168 & 89.4 & 100.0 & 93.7 \\
Focal-type AIP & & & 99.6 \\
Fit Asian & 68 & 73.1 & 100.0 & 95.6 \\
Fit revised HISORt & 73 & 78.5 & 100.0 & 97.3 \\
Fit ICDC & 79 & 84.9 & 100.0 & \\
\hline AIP, autoimmune pancreatitis; ICDC, International Consensus Diagnostic Criteria. & & \\
\hline
\end{tabular}

$100 \%$ and $93.7 \%$ (table 2). Using revised HISORt criteria, 158 patients $(84 \%)$ were diagnosed as definitive AIP. Among these 158 patients, the primary basis of diagnosis was diffuse type in 95 patients $(60.1 \%)$ and histology-based diagnosis in 31 patients $(19.6 \%)$. There were 30 patients who were deniable for AIP based on the revised HISORt criteria in this study. All of the patients with PC were deniable for AIP based on the revised HISORt criteria. The sensitivity, specificity and accuracy of the revised HISORt criteria were 84\%, $100 \%$ and $90.5 \%$ (table 2). Using Asian criteria, 162 patients $(86.2 \%)$ were diagnosed as AIP. In total 143 patients $(88.3 \%)$ were diagnosed based on imaging plus serology; 31 patients $(19.1 \%)$ were diagnosed based on histopathology and 126 patients $(77.8 \%)$ were diagnosed based on steroid treatment response. There were 26 patients who were deniable for AIP based on the Asian criteria

Table 3 Frequencies of levels 1 and 2 findings in ICDC for focal-type and diffuse-type AIP

\begin{tabular}{|c|c|c|}
\hline Features & $\begin{array}{l}\text { Focal-type } \\
\text { AIP }(n=93)\end{array}$ & $\begin{array}{l}\text { Diffuse-type } \\
\text { AIP }(n=95)\end{array}$ \\
\hline \multicolumn{3}{|l|}{ Ductal imaging } \\
\hline Level 1 & $0(\%)$ & $93(97.9 \%)$ \\
\hline Level 2 & $68(73.1 \%)$ & $0(0.0 \%)$ \\
\hline Level 1+2 & $68(73.1 \%)$ & $93(97.9 \%)$ \\
\hline Non-level 1, 2 & $25(26.9 \%)$ & $2(2.1 \%)$ \\
\hline \multicolumn{3}{|l|}{ Serology } \\
\hline Level 1 & $16 / 74(21.6 \%)$ & $31 / 88(35.2 \%)$ \\
\hline Level 2 & 25/74 (33.8\%) & $30 / 88(34.1 \%)$ \\
\hline Level $1+2$ & $41 / 74(55.4 \%)$ & $61 / 88(69.3 \%)$ \\
\hline Non-level 1, 2 & $33 / 74(44.6 \%)$ & $27 / 88(30.7 \%)$ \\
\hline \multicolumn{3}{|l|}{ OOI } \\
\hline Level 1 & $31(33.3 \%)$ & $32(\%) 33.7$ \\
\hline Level 2 & $45(48.4 \%)$ & $19(20.0 \%)$ \\
\hline Level $1+2$ & $76(81.7 \%)$ & $51(53.7 \%)$ \\
\hline Non-level 1, 2 & $17(11.3 \%)$ & $44(46.3 \%)$ \\
\hline \multicolumn{3}{|c|}{ Histology of the pancreas } \\
\hline Level 1 & $20(21.5 \%)$ & $8(8.4 \%)$ \\
\hline Level 2 & $2(2.2 \%)$ & $1(1.1 \%)$ \\
\hline Level $1+2$ & $22(23.7 \%)$ & $9(9.5 \%)$ \\
\hline Non-level 1, 2 & $71(76.3 \%)$ & $86(90.5 \%)$ \\
\hline
\end{tabular}

in this study. All of the patients with PC were deniable for AIP based on the Asian criteria. The sensitivity, specificity and accuracy of the revised HISORt criteria were $86.2 \%, 100 \%$ and $91.8 \%$ (table 2 ).

\section{Deniable cases on the basis of ICDC}

There were 20 patients who were deniable for ICDC in this study. All these 20 patients could be diagnosed by the Asian criteria. Among them, there were two patients who could be diagnosed both by the HISORt and Asian criteria. They included 6 and 14 cases with level 1 or 2 parenchymal imaging; 6 and 12 cases with level 1 or 2 ductal imaging; 9 cases with level 2 serology; 11 patients with level 1 OOI. There was one patient with a steroid treatment response. The most common factor which lead to deniable of ICDC or revised HISORt criteria was the serology criterion. There were 18 out of 20 patients who had autoantibodies which could be one of the items in the Asian serology criterion. The ICDC and HISORt only adapted IgG4 level alone as the serology criterion.

\section{ICDC criteria in focal-type and diffuse-type AIP}

The comparisons of frequencies of levels 1 and 2 findings in ICDC in focal-type and diffuse-type AIP are shown in table 3 . The frequencies of level 1 or 2 features in ductal imaging were significantly lower in focal-type AIP $(73.1 \%$ vs $97.9 \%, \mathrm{p}<0.001$, table 3$)$. The frequencies of level 1 or 2 features in serology were also lower in focal-type $(55.4 \%$ vs $69.3 \%, \mathrm{p}=0.075)$. The frequencies of any level of OOI in focal-type AIP were higher than those in diffuse-type AIP $(81.7 \%$ vs $53.7 \%, \mathrm{p}<0.0001)$. The frequencies of any histological evidence of LPSP in our focal-type AIP were higher than those in diffuse-type AIP (23.7\% vs $9.5 \%, p=0.01)$ in our study (table 3$)$.

\section{Sensitivity, specificity and accuracy of ICDC, revised HISORt criteria and Asian criteria}

The sensitivity, specificity and accuracy of ICDC were $84.9 \%, 100 \%$ and $93.8 \%$ (table 2). The sensitivity, specificity and accuracy of the revised HISORt criteria were $78.5 \%, 100 \%$ and $91.0 \%$ (table 2). The sensitivity, specificity and accuracy of the Asian criteria were $73.1 \%$, $100 \%$ and $88.8 \%$ (table 2). 
A

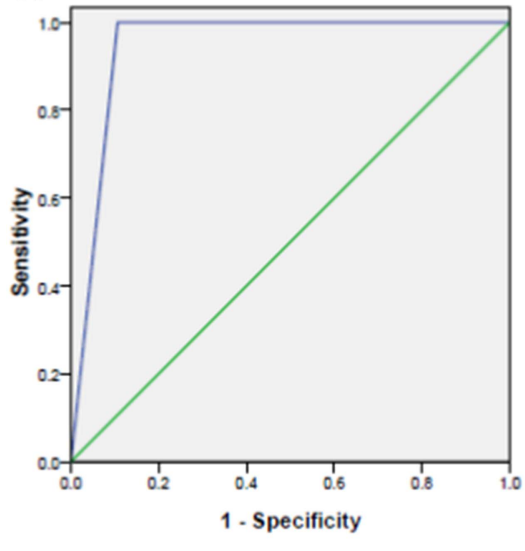

Area under ROC curve $=0.95(0.92-0.07)$
B

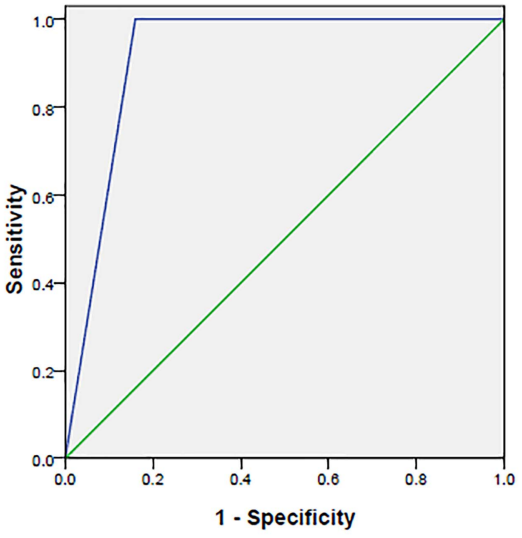

Area under ROC curve $=0.91$ (0.89-0.95)

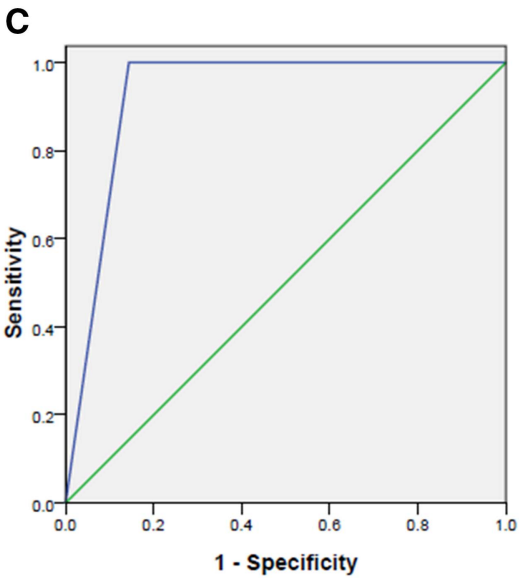

Area under $R O C$ curve $=0.93(0.89-0.96)$

Figure 1 Receiver-operator characteristic (ROC) curves of International Consensus Diagnostic Criteria (ICDC) and revised HISORT and Asian criteria in diagnosis of 188 patients with autoimmune pancreatitis from 130 patients. (A) ICDC diagnostic criteria. (B) Revised HISORT criteria. (C) Asian criteria.

The ROC was calculated in AIP (figure 1) and focaltype AIP (figure 2). The AUC was 0.95 (95\% CI 0.92 to 0.97 ) of ICDC (figure 1A), 0.91 (95\% CI 0.92 to 0.97 ) of the revised HISORt criteria (figure 1B) and 0.93 (95\% CI 0.92 to 0.97 ) of the Asian criteria (figure 1C). For focal-type AIP, the AUC was 0.93 (95\% CI 0.88 to 0.97 ) of ICDC (figure 2A), 0.89 (95\% CI 0.84 to 0.94 ) of the revised HISORt criteria (figure 2B) and 0.87 (95\% CI 0.81 to 0.92 ) of the Asian criteria (figure 2C).

\section{DISCUSSION}

The sensitivity, specificity and accuracy of ICDC for all AIP were $89.4 \%, 100 \%$ and $93.7 \%$. The sensitivity, specificity and accuracy of ICDC for focal-type AIP were $84.9 \%, 100 \%$ and $93.8 \%$. Among the three criteria, the sensitivity and accuracy of ICDC were the best compared with the revised HISORt and Asian criteria with the same specificity (table 2). There were 12 patients with deniable revised HISORt criteria who were diagnosed as AIP in ICDC (10 definite, 1 probable and 1 NOS of ICDC). There were 26 patients with deniable Asian criteria who were diagnosed as AIP in ICDC (11 definite, 14 probable and 1 NOS in ICDC). The ICDC showed higher sensitivity than the revised HISORt and Asian criteria. The reason why patients deniable for revised HISORt or Asian criteria but fit ICDC diagnosis was partially caused by the different definition of parenchymal imaging between these criteria. ICDC includes atypical imaging and classifies atypical imaging as S2 level in the parenchymal imaging criterion. That is one reason why ICDC had higher sensitivities in diagnosis of AIP in general. The wider range of imaging criteria improved the diagnostic sensitivity but did not decrease either the specificity or the accuracy of ICDC. All the three criteria showed high specificity (table 2). The ICDC showed higher accuracy than the Asian and revised HISORt criteria in our population. Recently, studies from the
A

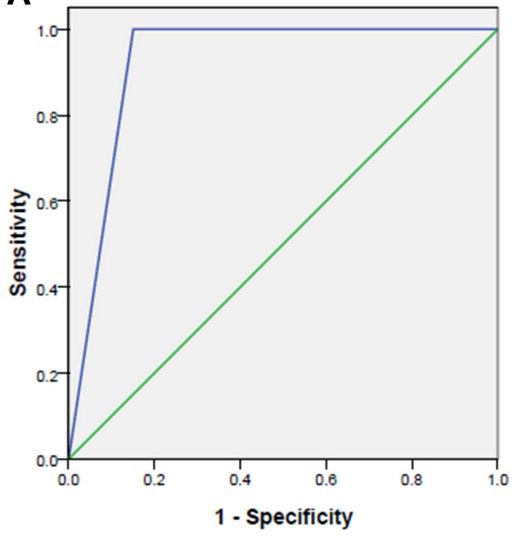

Area under ROC curve $=0.93(0.88-0.97)$

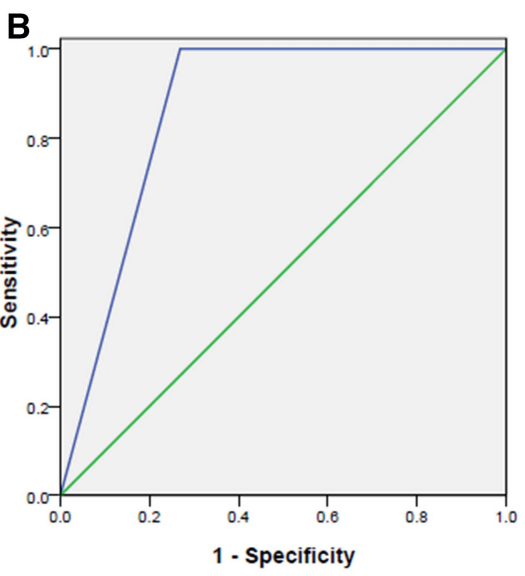

Area under ROC curve $=0.87(0.81-0.92)$

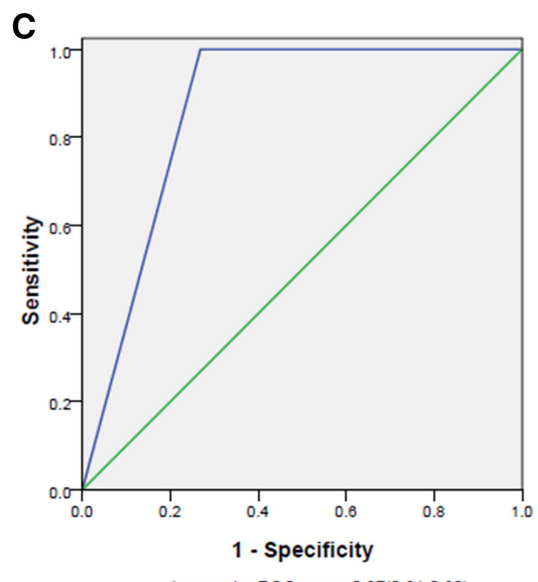

Area under ROC curve $=0.87(0.81-0.92$

Figure 2 Receiver-operator characteristic (ROC) curves of International Consensus Diagnostic Criteria (ICDC) and revised HISORT and Asian criteria in diagnosis of 93 patients with focal-type autoimmune pancreatitis from 130 patients with pancreatic cancer. (A) ICDC diagnostic criteria. (B) Revised HISORT criteria. (C) Asian criteria. 
Japanese population also demonstrated better accuracy of ICDC compared with other criteria, including the Japanese pancreatic society criteria. ${ }^{15-18}$ Although ICDC is considered to be superior to various other criteria, it seems to be too complicated to handle for clinicians. The Japanese have therefore proposed revised diagnostic criteria by the Japanese pancreatic society very recently. ${ }^{19}$ It would be interested to whether the performance could even be better or easlily to use in clinical practice compared to ICDC criteria. At this time moment, we did not have suitable simple amendment of ICDC of type I AIP which composed of a heterogenous population clinically in our country. With the advance of better understanding of the pathogenesis of the disease, to simplify the diagnostic criteria might be feasible and needed for clinicians.

In the ductal imaging criterion, 161 (92.5\%) of 188 patients with AIP and 13 (1\%) of 130 patients with PC were categorised as level 1 or 2, respectively. The specificity of ductal imaging is high. One of the reasons for this high specificity is that patients with marked upstream MPD dilation $(>5 \mathrm{~mm})$ were excluded from level 1 or 2 . In the present study, 28 of 31 patients with PC who showed focal MPD stricture were excluded from level 2 because of marked upstream MPD dilation. On the other hand, 27 (14.4\%) of 188 patients with AIP showed marked upstream MPD dilation. The frequency of any level 1 or 2 evidence in ductal imaging is close to the recently reported study $(7 / 62,11.3 \%)$ by Nishino et $a .^{20}$ Naitoh et $a l^{21}$ also reported that a maximal diameter of the upstream MPD less than $5 \mathrm{~mm}$ was an appropriate cut-off point to differentiate mass-forming AIP from PC. In our study, we also use the $5 \mathrm{~mm}$ as a cut-off point to differentiate focal-type AIP and PC. Therefore, we consider that a $5 \mathrm{~mm}$ diameter of the upstream MPD is appropriate to discriminate AIP from PC. In the present study, four patients with PC fulfilled the level 1 serological criterion. Marked upstream MPD dilation $(5 \mathrm{~mm})$ was observed in these patients. If this exclusion criterion (marked upstream MPD) did not exist, this patient would have fulfilled the level 2 criterion for ductal imaging, and we would have misdiagnosed these four patients with PC as definitive type 1 AIP under ICDC. Therefore, we consider that this exclusion criterion of ductal imaging is useful for excluding PC.

The value of serum IgG4 as a serological marker of AIP was first established in 2001.22 ${ }^{23}$ Hamano et $a t^{23}$ reported that sensitivity and specificity for differentiating AIP from PC were $90.2 \%$ and $97.5 \%$. In the present study, the sensitivity and specificity of serum IgG4 $(>140 \mathrm{mg} / \mathrm{dL})$ were $79.6 \%$ and $92.6 \%$, respectively. The cause of the wide range of sensitivity in the reported series might be caused by the combined analysis of types 1 and 2 in these studies. The distribution between types 1 and 2 AIP might affect the value of IgG4. Ghazale et at ${ }^{4}$ reported that the sensitivity of elevated serum IgG4 $(>140 \mathrm{mg} / \mathrm{dL})$ for PC was $10 \%$, and that of twofold elevation (level 1 ICDC serology criteria) was $1 \%$. Our present study showed that the sensitivity and specificity of twofold elevation of serum IgG4 were $36.7 \%$ and $95.2 \%$ in our type 1 AIP. Serum IgG4 is the only used serology marker in ICDC. In the Asian criteria, they adapted $\operatorname{IgG}, \operatorname{IgG} 4$ and the presence of an autoantibody as serological criteria. In our patients with type 1 AIP, if we add the presence of an autoantibody also as a surrogate marker in serology, all of the patients deniable for ICDC could be diagnosed by this modification.

IgG4-related disease (IgG4-RD) is a new disease entity characterised by elevated serum IgG4 concentration and/or tissue infiltration by IgG4-positive cells. ${ }^{24}$ Type 1 AIP is regarded as a part (pancreatic manifestation) of IgG4-RDs. In the present study, level 1 or 2 OOI was observed in $127(67.6 \%)$ of the patients with AIP and in none of those with PC. Therefore, this OOI criterion has high specificity for type 1 AIP.

In the present study, all resection specimens (27 patients) fulfilled the level 1 criterion. One of four biopsied specimens guided by CT fulfilled level 1, with the rest diagnosed as level 2. The present findings suggest that obtaining histopathological evidence of type 1 AIP by a biopsied specimen is difficult. EUS-FNA was considered useful for the differentiation from PC but might be insufficient for tissue collection to diagnosis as level 1. Kanno et $a l^{25}$ reported that 14 and 6 patients, respectively, of 25 patients were judged to have levels 1 and 2 histological findings by trucut biopsy under endoscopic ultrasound (EUS) guidance. We did not perform EUS-trucut biopsy in this study because to the needle is not available and the cost is not covered by our health insurance. Further studies for the feasibility and necessarily of EUS-FNA in diagnosing type 1 AIP in ICDC histological criteria need to be studied.

Diffuse and focal enlargement of the pancreas is a characteristic feature of AIP in parenchymal imaging. ICDC included patients with atypical parenchymal imaging as level 2. For diffuse-type AIP, the diagnostic accuracy of ICDC and Asian and HISORt criteria was over $95 \%$, much better than that in focal-type AIP. This observation is reasonable for us to understand because it is very rare to have PC involving the whole pancreas in clinical practice. The diagnosis sensitivity in diffuse-type AIP was also higher than those in focal-type AIP in these three diagnostic criteria (table 2). In diffuse-type AIP, the Asian criteria were most sensitive with a sensitivity of $98.9 \%$, followed by ICDC $(93.7 \%)$ and the HISORt criteria $(89.5 \%)$. There were four patients with diffuse-type AIP who did not have elevated was the least sensitive criteria in the diagnosis of diffuse type with sensitivity only $89.5 \%$. The four diffuse-type AIP patients were deniable for HISORt but fit ICDC NOS owing to these four patients did not have any collateral evidence. These four patients could be diagnosed by Asian criteria as they have presence of autoantibodies which made they fit the serology criteria in Asian diagnosis setting but not fit the serology criteria in ICDC and HISORt criteria. These four patients had received steroid response as the initial 
treatment and they all had disease relapse in their follow-up. The increase of sensitivity in the Asian criteria in diagnosing diffuse-type AIP is relevant to the wide range of definition in the serology criterion (IgG, IgG4 or/and autoantibodies), compared to the use of IgG4 alone as the serology criterion in HISORt and ICDC.

It is a greater challenge to differentiate focal-type AIP with PC, compared to differentiate diffuse-type AIP from PC. In this study, we have 93 patients with focal-type AIP. The ductal imaging, serology and OOI were different from those for diffuse-type AIP. Focal-type AIP had less frequency of level 1 or 2 presentations of ductal imaging and serology. In contrast, focal-type AIP had higher frequencies of level 1 or 2 presentations of OOI. These clinical observations remind us that the collateral evidence of AIP in focal-type AIP is mostly in OOI, but not serology. Detailed physical examination and history taking and imaging interpretation imaging other than pancreas could give some hint to increase the sensitivity of diagnosis of focal-type AIP.

In focal-type of AIP, the diagnostic sensitivity of ICDC $(84.9 \%)$ was higher than those of the revised HISORt $(78.5 \%)$ and Asian criteria $(73.1 \%)$. There were 14 (15.1\%) patients with focal-type AIP deniable for the ICDC criteria. All these 14 patients could be diagnosed by the Asian criteria. There were 12 patients with the presence of autoantibodies and 9 patients with a serum IgG4 level above $140 \mathrm{mg} / \mathrm{dL}$. Among them, there were 12 patients with level 2 criteria and 2 patients without level 1 or 2 criteria in ductal imaging. In OOI, there were nine patients with level 2 criteria and five patients without level 1 or 2 criteria. All the patients with focaltype AIP deniable for the Asian criteria could be diagnosed by the ICDC criteria.

In conclusion, ICDC shows high sensitivity, specificity and accuracy in the diagnosis of type 1 AIP. In focal-type AIP, ICDC is still the best in sensitivity, specificity and accuracy. The diagnostic sensitivity in focal-type AIP is not as good as diffuse-type AIP in all the three adapted criteria (ICDC and Asian and revised HISORt criteria). How to improve the sensitivity of diagnosis of focal-type AIP is the issue that needs to be resolved in the future.

\section{Author affiliations}

${ }^{1}$ Department of Internal Medicine, National Taiwan University Hospital, College of Medicine, National Taiwan University, Taipei, Taiwan

${ }^{2}$ Department of Radiology, National Taiwan University Hospital, College of Medicine, National Taiwan University, Taipei, Taiwan

${ }^{3}$ Department of Laboratory Medicine, National Taiwan University Hospital, College of Medicine, National Taiwan University, Taipei, Taiwan ${ }^{4}$ Department of Surgery, National Taiwan University Hospital, College of Medicine, National Taiwan University, Taipei, Taiwan

Acknowledgements The authors are thankful for the help of the Department of Laboratory Medicine in the National Taiwan University Hospital for technical support and analysis of serum IgG4. They express their deep sense of gratitude to all the individuals who agreed to participate in the study.

Contributors MC-C and YT-C had full access to the data and take responsibility for the integrity of the data and the accuracy of the data analysis. MC-C, PC-L, IS-J, CY-Y, YW-T,SC-W, JM-W and YT-C were involved in the conception and design, or analysis and interpretation of data. MC-C and YT-C were involved in the drafting of the article or its critical revision for important intellectual content and final approval of the version to be published. MC-C, YT-C and JM-W obtained funding and provided administrative, technical or material support. YT-C was involved in study supervision and had the final responsibility for the decision to submit for publication.

Funding National Science Council, Taiwan (NSC 94-2314-B-002-272) and NTUH (National Taiwan University Hospital)-95-M-22, NTUH-97-M-1001 and NTUH-9-M-1227; NSC 102-2321-B-002-083-; MOHW103-TD-B-111-04; Liver Disease Prevention and Treatment Research Foundation and New Century Health Care Promotion Foundation.

Competing interests None.

\section{Patient consent Obtained.}

Ethics approval The study protocol was approved by the institutional review board of the National Taiwan University Hospital.

Provenance and peer review Not commissioned; externally peer reviewed.

Data sharing statement No additional data are available.

Open Access This is an Open Access article distributed in accordance with the Creative Commons Attribution Non Commercial (CC BY-NC 4.0) license, which permits others to distribute, remix, adapt, build upon this work noncommercially, and license their derivative works on different terms, provided the original work is properly cited and the use is non-commercial. See: http:// creativecommons.org/licenses/by-nc/4.0/

\section{REFERENCES}

1. Yoshida K, Toki F, Takeuchi T, et al. Chronic pancreatitis caused by an autoimmune abnormality. Proposal of the concept of autoimmune pancreatitis. Dig Dis Sci 1995;40:1561-8.

2. Kamisawa T, Chari ST, Lerch MM, et al. Recent advances in autoimmune pancreatitis: type 1 and type 2. Gut 2013;62:1373-80.

3. Kamisawa T, Imai M, Yui Chen $\mathrm{P}$, et al. Strategy for differentiating autoimmune pancreatitis from pancreatic cancer. Pancreas 2008;37: e62-7.

4. Ghazale A, Chari ST, Smyrk TC, et al. Value of serum IgG4 in the diagnosis of autoimmune pancreatitis and in distinguishing it from pancreatic cancer. Am J Gastroenterol 2007;102:1646-53.

5. Morselli-Labate AM, Pezzilli R. Usefulness of serum IgG4 in the diagnosis and follow up of autoimmune pancreatitis: a systematic literature review and meta-analysis. $J$ Gastroenterol Hepatol 2009;24:15-36.

6. Kamisawa T, Okazaki K, Kawa S. Diagnostic criteria for autoimmune pancreatitis in Japan. World J Gastroenterol 2008;14:4992-4.

7. Okazaki K, Kawa S, Kamisawa T, et al. Clinical diagnostic criteria of autoimmune pancreatitis: revised proposal. $J$ Gastroenterol 2006;41:626-31.

8. Otsuki M, Chung JB, Okazaki K, et al. Asian diagnostic criteria for autoimmune pancreatitis: consensus of the Japan-Korea Symposium on Autoimmune Pancreatitis. J Gastroenterol 2008:43:403-8.

9. Chari ST, Smyrk TC, Levy MJ, et al. Diagnosis of autoimmune pancreatitis: the Mayo Clinic experience. Clin Gastroenterol Hepatol 2006;4:1010-16; quiz 934.

10. Shimosegawa $T$, Chari ST, Frulloni $L$, et al. International consensus diagnostic criteria for autoimmune pancreatitis: guidelines of the International Association of Pancreatology. Pancreas 2011:40:352-8.

11. Chang MC, Jan IS, Liang PC, et al. PRSS1 but not SPINK1 variants increase the risk of type 1 autoimmune pancreatitis. J Gastroenterol Hepatol 9 Jun 2014. Epub ahead of print.

12. Chang MC, Chang YT, Tien YW, et al. T-cell regulatory gene CTLA-4 polymorphism/haplotype association with autoimmune pancreatitis. Clin Chem 2007;53:1700-5.

13. Chang MC, Chang YT, Wei SC, et al. Autoimmune pancreatitis associated with high prevalence of gastric ulcer independent of Helicobacter pylori infection status. Pancreas 2009;38:442-6.

14. Haaga JR, Alfidi RJ, Zelch MG, et al. Computed tomography of the pancreas. Radiology 1976;120:589-95.

15. Maruyama M, Watanabe T, Kanai K, et al. International Consensus Diagnostic Criteria for autoimmune pancreatitis and its Japanese amendment have improved diagnostic ability over existing criteria Gastroenterol Res Pract 2013;2013:456965. 
16. Naitoh I, Nakazawa T, Hayashi K, et al. Clinical evaluation of international consensus diagnostic criteria for type 1 autoimmune pancreatitis in comparison with Japanese diagnostic criteria 2011. Pancreas 2013:42:1238-44.

17. Tabata T, Kamisawa T, Kuruma $\mathrm{S}$, et al. Capability and limitations of recent diagnostic criteria for autoimmune pancreatitis. Int $J$ Rheumatol 2013;2013:465428.

18. Sumimoto K, Uchida K, Mitsuyama T, et al. A proposal of a diagnostic algorithm with validation of International Consensus Diagnostic Criteria for autoimmune pancreatitis in a Japanese cohort. Pancreatology 2013;13:230-7.

19. Kawa S, Okazaki K, Kamisawa T, et al. Amendment of the Japanese Consensus Guidelines for Autoimmune Pancreatitis, 2013 II. Extrapancreatic lesions, differential diagnosis. J Gastroentero 2014:49:765-84.

20. Nishino $\mathrm{T}$, Oyama $\mathrm{H}$, Toki $\mathrm{F}$, et al. Differentiation between autoimmune pancreatitis and pancreatic carcinoma based on endoscopic retrograde cholangiopancreatography findings. $J$ Gastroenterol 2010;45:988-96.

21. Naitoh I, Nakazawa T, Hayashi K, et al. Clinical differences between mass-forming autoimmune pancreatitis and pancreatic cancer. Scand J Gastroenterol 2012;47:607-13.

22. Aithal GP, Breslin NP, Gumustop B. High serum IgG4 concentrations in patients with sclerosing pancreatitis. N Engl J Med 2001;345:147-8.

23. Hamano $\mathrm{H}$, Kawa $\mathrm{S}$, Horiuchi $\mathrm{A}$, et al. High serum IgG4 concentrations in patients with sclerosing pancreatitis. $N$ Engl $J$ Med 2001;344:732-8.

24. Kamisawa T, Funata N, Hayashi $\mathrm{Y}$, et al. A new clinicopathological entity of IgG4-related autoimmune disease. J Gastroenterol 2003;38:982-4

25. Kanno A, Ishida K, Hamada S, et al. Diagnosis of autoimmune pancreatitis by EUS-FNA by using a 22-gauge needle based on the International Consensus Diagnostic Criteria. Gastrointest Endosc 2012;76:594-602. 